\title{
Emotional Intelligence for Healthcare
}

\author{
Joseph Farmer ${ }^{1}$, Mita Mistry ${ }^{2}$, Ashok Kumar Jainer ${ }^{1}$ \\ 1 Coventry \& Warwickshire NHS Partnership Trust \\ 2 British Acupuncture Council \\ Correspondence to joseph.farmer@nhs.net
}

\author{
Key words \\ Emotional intelligence, empathy, healthcare professionals
}

Cite as: Farmer J, Mistry M, Winchester M, Kumar A. Emotional Intelligence for Doctors. Sushruta J Health

Policy \& Opin 2020 vol 3:issue 2 epub 24.02.2020 DOI: 10.38192/13.1.8

What is Emotional Intelligence?

Emotional intelligence is a term popularised by the American Psychologist Daniel Goleman ${ }^{[1]}$. It is the ability to recognise, understand and control one's emotions and consider the impact these have on oneself and others around them, particularly in the context of social interaction. Emotional intelligence can therefore allow for the formation of strong friendships, effective leadership abilities, high performing teams, and is usually identified in high achieving individuals ${ }^{[2]}$.

It is broadly recognised that emotional intelligence is comprised of four main components:

- Self-awareness - this represents one's ability to recognise one's own emotions and responses to a particular situation. This can also extend to recognising one's own strengths and weaknesses, cultural influences, biases, values, and how this impacts and influences others.

- Self-management - refers to the ability to control one's emotional response to a particular situation, allowing for healthy, nondisruptive interactions. In combination with self-awareness, the ability to temper and alter a response in a social setting can prevent escalating negative interactions and allow for healthy relationships to continue.

- Social awareness - refers to the ability to recognise and act on the emotional responses of others in an empathic way. Reacting to these can facilitate communication, enhance relationships and support others. Social awareness can also extend to recognising power dynamics within a team, or organisation.

- Relationship management - indicates the ability to healthily and successfully maintain social relationships, interact with others in a productive way, work in teams, and even lead and inspire those around them.

Emotional intelligence is linked to intellectual intelligence (IQ), but operates as a separate system ${ }^{[3]}$. This was demonstrated by Damasio, who showed that impairment to the prefrontal cortex impairs emotional response and rational decision making, while leaving intellectual and cognitive function intact $^{[4]}$. It is generally felt that IQ is something fixed, determined in development by the interplay of genetics and environment, whereas emotional intelligence is more malleable and can be taught and improved.

\section{Why is Emotional Intelligence Important?}

Developing skills in emotional intelligence has numerous benefits. Prati et al describe how a leader with higher emotional intelligence directly correlates to higher team effectiveness ${ }^{[5]}$. A leader who can recognise the emotional responses of those around them, navigate how these team-members interact and communicate, and predict responses of those around them, can better motivate that team to perform. A leader with high emotional intelligence can simultaneously create an environment of empowerment and support, resulting in team members who show higher self-motivation, and a conscientious responsibility to want to achieve the 
best for that team. In business this can mean greater profits, high employee satisfaction and worker retention. It can create teams that innovate, perform highly, and produce high quality work. In medicine this can mean better performing teams that are providing better care for their patients.

Emotional intelligence also plays a key role in dealing with conflict ${ }^{[6]}$. Being aware of your own emotions in a situation allows you to control them, identify the best approach based on the perceived needs of others and how your response interplays with their emotions, in order to proceed in a productive manner when working with others. Utilising this approach effectively can often avoid conflict. If there has been conflict, developing high emotional intelligence can allow for greater success at conflict resolution. A study by Başoğul and Özgür demonstrated the improved outcomes between nurses when the nurse in charge allocated to mediate the conflict, scored higher on their emotional intelligence scales ${ }^{[7]}$.

A study by Shahid et al ${ }^{[8]}$ reviewed how developing emotional intelligence may benefit doctors. They reported that inclusion of an educational intervention to help improve emotional intelligence "may improve stress management skills, promote wellness and prevent burnout in resident physicians". With reported levels of burnout amongst doctors increasing, they proposed the benefits of emotional intelligence would be widespread and significant. Recognising your own emotions in response to stressful environments, in this case in a clinical setting, can allow you to develop healthier coping mechanisms and prevent impact on inter-personal relationships and patient care. The study demonstrated significant improvements in emotional intelligence after an educational course, evidencing that this is a skill that can be learned and improved.

A doctor with higher levels of emotional intelligence can lead to improved patient satisfaction. An observational study by Hui-Ching Weng utilised selfsurveys across doctors and patients in an out-patient setting and found that doctors with higher emotional intelligence correlated to increased patient satisfaction ${ }^{[9]}$. One of the difficult tasks of any patient interaction is reaching a level of understanding with a patient that best allows you to mutually agree an appropriate management plan. We have already discussed how emotional intelligence allows one to regulate their own emotions, control them in their responses, and perceive and react to others' emotions. It makes sense that this would be beneficial when it comes to doing this with a patient, as well as when acting in a team. Conflict resolution skills can also be utilised here with patients and family and allow for far greater outcomes in those with high emotional intelligence.

It has been considered whether emotional intelligence should be included as part of a selection process for doctors, given its numerous and recognised benefits ${ }^{[10]}$. Peggy Wagner discussed the potential to not only be able to identify those individuals with sufficient IQ required to manage the theoretical aspects of the profession, but also the emotional intelligence to be able to supplement this. They argue the role of a physician extends beyond book learning to handling difficult patient interactions, working within many different teams, usually with leadership roles, all of which require emotional intelligence. However, they felt that it is not a reliable way to select candidates, and instead, should form part of the educational experience after successful application.

\section{Conclusions}

There is an increasingly pressing need for doctors to understand their own emotional reactions to what they experience in their practice. Doctors are exposed to a variety of traumatic situations from distressed patients and relatives to witnessing death. Doctors are human and most, if not all humans, will absorb some of the emotional burden even in their subconscious.

The experience of doctors practicing in the current climate is stressful with increasing regulations aimed at improving quality of care. Therefore, it is not surprising they are often overwhelmed in a tug-of-war between administrators, staff, colleagues and most importantly, patients. With many doctors experiencing burnout, questions arise regarding their mental health and work-life balance.

Improving emotional intelligence can help doctors become more aware of the burden, allow them to regulate their response and develop healthy coping mechanisms. This can contribute towards improving doctor's overall health. Further to this, recognising the impact of emotion as an individual opens a pathway to respond to these traumatic situations with patients optimally and address personal work-life balance issues.

Indeed, in reality it can be challenging to be both scientifically objective, clinically competent and 
empathetic towards patients, but what about empathy towards ourselves? Perhaps the current "ideal" image of doctors is that of a clinical, rational, skilful, yet somewhat emotionally detached persona. To be emotionally expressive often is perceived to equate to unprofessionalism. It is these very images which prevent doctors from engaging with their patients emotionally and with their own feelings. Engaging with our own feelings forms the foundation of empathy derived from a level of emotional intelligence.

Doctors should be supported with this emotional burden in the healthcare environment. Empathy and emotional intelligence should not only be expected from doctors but should be actively promoted, assisted and cultivated in the medical profession. Practices like emotional intelligence and mindfulness provide vehicles for realistic long-lasting solutions which will be imperative given the increasing pressures doctors will continue to face. Institutional support and educational intervention has been proven to help and should be utilised at every possible opportunity.

\section{References}

1. Goleman, D. (1996). Emotional Intelligence. Why It Can Matter More than IQ.Learning, 24(6), 49-50.

2. Brian McMullen. Emotional intelligence. BMJ 2003;326:030218

3. McMullen B. Cognitive intelligence. Student BMJ2002;10:458-9.

4. Damasio A. Descartes error. London: Picador, 1995:34-51

5. L. Melita Prati, Ceasar Douglas, Gerald R. Ferris, Anthony P. Ammeter, M. Ronald Buckley. Emotional intelligence, leadership effectiveness, and team outcomes. The International Journal of Organizational Analysis, Vol 11, No. 1, 2003, pp.21-40.

6. Chan JC, Sit EN, Lau WM. Conflict management styles, emotional intelligence and implicit theories of personality of nursing students: a cross-sectional study. Nurse Educ Today. 2014 Jun;34(6):934-9. doi: 10.1016/j.nedt.2013.10.012.

7. Başoğul, Özgür. Role of Emotional Intelligence in Conflict Management Strategies of Nurses. Asian Nurs Res (Korean Soc Nurs Sci). 2016 Sep;10(3):228-233. doi:
8. Ramzan Shahid, Jerold Stirling, William Adams. Promoting wellness and stress management in residents through emotional intelligence training. Advances in Medical Education and Practice, 2018; Volume 9: 681 DOI: 10.2147/AMEP.S175299

9. Hui-Ching Weng Chao-Ming Hung Yi-Tien Liu Yu-Jen Cheng Cheng-Yo Yen Chi-Chang Chang Chih-Kun Huang. Associations between emotional intelligence and doctor burnout, job satisfaction and patient satisfaction. Medical Education Volume 45, Issue 8. First published:14 July 2011. https://doi.org/10.1111/i.13652923.2011.03985.x

10. Peggy J. Wagner, PhD. Does High El (Emotional Intelligence) Make Better Doctors? Virtual Mentor. 2006;8(7):477-479. doi: 10.1001/virtualmentor.2006.8.7.oped2-0607.

10.1016/j.anr.2016.07.002. Epub 2016 Jul 19. 